\title{
Esthétique du régionalisme
}

Aesthetics of regionalism

\section{Thierry Glon}

\section{CpenEdition}

\section{Journals}

Édition électronique

URL : https://journals.openedition.org//bl/1037

DOl : $10.4000 / \mathrm{lbl} .1037$

ISSN : 2727-9383

\section{Éditeur}

Université de Bretagne Occidentale - UBO

\section{Édition imprimée}

Date de publication : 1 juin 2015

Pagination : 43-54

ISBN : 979-10-92331-16-5

ISSN : 1270-2412

\section{Référence électronique}

Thierry Glon, « Esthétique du régionalisme », La Bretagne Linguistique [En ligne], 19 | 2015, mis en ligne le 01 mai 2021, consulté le 22 mai 2021. URL : http://journals.openedition.org//bl/1037 ; DOI : https:// doi.org/10.4000/lbl.1037

\section{cc) (7)}

La Bretagne Linguistique est mise à disposition selon les termes de la Licence Creative Commons Attribution 4.0 International. 


\section{Thierry GLON*}

\section{Esthétique du régionalisme}

' exclusion de la littérature régionaliste hors des histoires litté-
raires dit assez le discrédit de ce qui fut pourtant un immense conti-
nent dans l'édition française, entre 1850 et $1950^{1}$, et qui continue à
vendre malgré son faible prestige. Ce discrédit va sans dire : il est
si unanime que les défauts qu'on lui impute n'ont pas même besoin
d'être affirmés. On les devine aisément. D'une part la littérature
régionaliste a assurément figé des stéréotypes régionaux. D'autre
part elle a limité ses ambitions au clocher du village alors que la
littérature française est créditée d'une vocation universelle ${ }^{2}$. De fait,
la littérature régionaliste s'est probablement mise hors jeu au mo-
ment où, dans la lignée de Baudelaire, la modernité littéraire séparait
fortement l'éthique de l'esthétique : la littérature régionaliste mettait
au contraire l'accent sur des objets spécifiques (les classes muettes

* Maître de conférences, Université de Nantes.

1. A.-M. Thiesse distingue deux périodes : le tournant du siècle où s'élabore une décentralisation littéraire qui oppose la province et Paris (les auteurs sont nés entre 1865-1880). Puis un temps de «successeurs» qui triomphent dans l'entredeux-guerres (et sous le régime de Vichy qui encense les écrivains ruraux), raflant les prix Goncourt, concurrençant les succès du roman de mœurs dans les revues et les librairies.

2. C'est la thèse du critique littéraire André Billy, dans un article de 1946 intitulé «Est-ce la fin du régionalisme ?» (Figaro littéraire, samedi 29 juin 1946). 
et laborieuses, à l'exception de la classe ouvrière) et sur des thèmes moraux, opposés à ceux d'une littérature parisienne réputée friande de l'alcôve ou du crime. C'est sans doute ce critère d'ambition esthétique qui fait qu'on ne compte pas Madame Bovary parmi les romans régionalistes alors que ce livre n'excède pas un cercle de trente kilomètres de diamètre autour de Rouen.

À cela s'ajoute sans doute un reproche de médiocrité littéraire. On peine à trouver des manifestes où s'exprimerait une esthétique régionaliste. On se croit donc fondé à penser que ces auteurs se soumettaient à l'air du temps, ce qui fait que Paul Vernois et même A-M. Thiesse lisent implicitement ces textes comme des copies, forcément plus pâles, des grandes esthétiques : du Parnasse (lorsqu'ils sont écrits avec l'amour du mot rare et des audaces versifiées), de l'idylle pastorale (dans une lignée qui irait de Théocrite et Longus jusqu'à George Sand) ou du réalisme (avec deux textes qui seraient fondateurs : Les Paysans de Balzac et La Terre de Zola). De fait, les écrivains régionalistes paraissent davantage occupés à tisser un réseau littéraire et à mettre en anthologie leurs écrits pour faire nombre, plutôt qu'à affirmer des choix esthétiques. Lorsqu'on lit Les Littératures provinciales (1907) de Jean Charles-Brun, lorsqu'on parcourt la compilation d'Adolphe Van Bever qui est publiée autour de 1910 (Les poètes du terroir du XVe au XXe siècle, C. Delagrave, Paris, 4 vol.) ou lorsqu'on se tourne vers l'Esthétique régionaliste de Mafféo-Charles Poinsot (1911), on repère à peu près la même structure : une réflexion introductive (un peu plus précise chez Jean Charles-Brun), puis une liste de noms d'auteurs et une anthologie. Comme l'écrit Anne-Marie Thiesse, "l'esthétique du régionalisme littéraire n'est en fait jamais énoncée par ses laudateurs, qu'à travers une longue énumération d'œuvres, sans que les critères de sélection soient jamais explicités ${ }^{3}$.» Par exemple, pour Jean Charles-Brun, l'essentiel est une littérature toujours «vivifiée par le contact avec le sol», «un art local, un art particulier et approprié». C'est-à-dire que le programme esthétique se réduit à l'enracinement personnel et intellectuel : vivre ici et étudier la tradition.

La description du champ littéraire dans le cadre conceptuel de

3. A.-M. THIESSE, Écrire la France : le mouvement littéraire régionaliste de langue française entre la Belle Epoque et la Libération, Paris, PUF, 1991, p. 105. 
Pierre Bourdieu a éclairé la pratique régionaliste et Anne-Marie Thiesse y a décrit de façon lumineuse une stratégie de petits bourgeois mal introduits dans le milieu littéraire pour contourner leur indigence en capital culturel : le régionalisme leur fut un moyen d'être publiés, au risque de se trouver relégués à vie dans une catégorie subalterne. Dans un temps où, à la différence de la ville où le péril social s'est déplacé, les campagnes représentent un sanctuaire où l'on imagine que la vie est saine et que le travail n'est pas aliénation mais accomplissement, ces écrivains se valorisent comme représentants d'un peuple mythique (le peuple réel étant celui qu'ils ont réussi à quitter à force d'études) : "Contre le capital culturel et social hérité de ceux qui ont "naturellement" leur place dans les salons lettrés et dans les positions dominantes du champ littéraire, les "non-héritiers" invoquent le seul capital hérité dont ils puissent se prévaloir : l'enracinement dans un terroir, dans le Peuple, dans une culture qui serait spontanément issue du Sol et de la Tradition ${ }^{4} »$.

Si l'on souhaite donner à la production régionaliste une chance de servir à autre chose qu'à illustrer le fonctionnement du champ littéraire français et d'avoir encore aujourd'hui quelque chose d'esthétique à nous dire, alors c'est vers un auteur qui précède ce mouvement (et qui lui a servi souvent de repoussoir) qu'il faut se tourner. George Sand en a énoncé les problèmes dans les courtes préfaces de ses romans berrichons. Outre le choix de l'idéalisation qui ne nous concernera pas ici, la notice de La Mare au Diable, en 1845, affirme la croyance que le paysan aurait un sens inné du beau mais incommunicable par manque d'instruction ou par l'abrutissement lié au travail et à la misère. George Sand s'y demande donc comment faire parler la classe muette. Comment se faire truchement entre Paris et la paysannerie ? C'est dans l'avant-propos de François le Champi que l'auteur tente de répondre sans sous-estimer la complexité de la question. George Sand affirme d'abord la nécessité de l'empathie : entre l'écrivain qui parle et le paysan qui sent il y a un terrain d'entente qui est le sentiment - «Pour parler le langage que tu adoptes, je te répondrai qu'entre la connaissance et la sensation, le rapport c'est le

4. Michel Espagne, Michael Werner, «Qu'est-ce qu'une littérature nationale ? Approches pour une théorie interculturelle du champ littéraire», Philologiques, tome III, Paris, éditions MSH, 1994. 
sentiment». Puis elle énonce une formule d'ambiguité qui inaugure l'immense travail régionaliste d'effacement de l'écrivain : l'auteur rustique doit adopter une écriture de compromis entre la «langue de l'Académie» et le dialecte qui aurait le défaut d'être incompréhensible mais qui serait supérieur "pour rendre tout un ordre d'émotions, de sentiments et de pensées ». Il faudrait raconter comme si l'on avait à sa droite un Parisien, et à sa gauche un paysan :

«Tiens, commence, raconte-moi l'histoire du Champi, non pas telle que je l'ai entendue avec toi. C'était un chef-d'œuvre de narration pour nos esprits et pour nos oreilles du terroir. Mais raconte-la-moi comme si tu avais à ta droite un Parisien parlant la langue moderne, et à ta gauche un paysan devant lequel tu ne voudrais pas dire une phrase, un mot où il ne pourrait pas pénétrer. Ainsi tu dois parler clairement pour le Parisien, naïvement pour le paysan. L'un te reprochera de manquer de couleur, l'autre d'élégance. »

La littérature régionaliste pratique donc souvent de laborieux subterfuges pour assurer une délégation de parole et pour légitimer son narrateur comme un truchement qui puisse jouxter le peuple sans pour autant cesser d'écrire comme un homme cultivé, et les auteurs ont souvent recours à d'improbables chaînes de témoins, une noria de on-dit. Dans François le Champi, La petite Fadette et Les Maîtres sonneurs, c'est la servante du curé, relayée par le chanvreur Depardieu qui sont gardiens d'une parole que l'auteur ne ferait que traduire. Mais cette écriture à deux intermédiaires est souvent plus complexe : le récit de Fille de fraudeurs d'Anatole Le Braz est porté par un brigadier qui raconte une anecdote à son jeune lieutenant qui la rapporte lui-même à un ultime officier des douanes qui a l'aisance de plume d'un homme de lettres. L'Îlienne ou Le Cavalier de la mer accumulent les couches de narrateurs, ce qui alourdit ces romans beaux et troublants de Jeanne Nabert. Dans Le Cavalier de la mer la vraisemblance est douteusement assurée par un «Monsieur» âgé qui attend son train et que la pluie chasse dans une grande maison dont la servante Catherine Dall lui raconte l'histoire. Peut-être le voyageur anonyme prend-il en charge la langue poétique tandis que les renseignements ethnographiques ainsi que le ton vigoureux reviennent à Catherine Dall - mais il est bien hasardeux de les départager. 
Enfin, à propos de cette situation ambiguë du narrateur, George Sand assume une critique qui concerne Jeanne, son échec littéraire de 1844. Selon elle, cet échec s'expliquerait par la rupture de ton entre les paroles toutes prosaïques de son personnage et la description épique que l'auteur en fait :

«Tu mets dans sa bouche des paroles qu'à la rigueur elle peut dire. Mais toi romancier, qui veux faire partager à tes lecteurs l'attrait que tu éprouves à peindre ce type, tu la compares à une druidesse, à Jeanne d'Arc, que sais-je ? Ton sentiment et ton langage font avec les siens un effet disparate comme la rencontre de tons criards. »

Or c'est cette dimension disparate et rhapsodique qui me parait essentielle dans les œuvres régionalistes. Je fais l'hypothèse que les écrivains y adoptent résolument « la rencontre de tons criards », c'està-dire une esthétique de la rupture de ton qu'ils ne théorisent pourtant jamais systématiquement, si ce n'est sous la forme de bribes dans des lettres ou des commentaires. Cette esthétique rhapsodique est rendue possible par l'atténuation des hiérarchies entre les tons au cours du $\mathrm{XIX}^{\mathrm{e}}$ siècle et, particulièrement, par le prestige nouveau du roman, genre du mélange et du dialogisme - est-il besoin de dire que Les Misérables rassemble sublime, grotesque, épique, idylle, réflexion philosophique et réalisme social?

Les œuvres régionalistes organisent une disparate séquentielle, comme si l'unité de ton leur était impossible. Elles dérivent entre grandeur épique, humble pastorale ou réalisme truculent. L'occitan Ferdinand Fabre, auteur du roman Le Chevrier qui a connu un certain succès en 1867, mêle ainsi l'épopée, le réalisme balzacien et la pastorale au sein de laquelle il parvient paradoxalement à insérer une gauloiserie vigoureuse. On passe ainsi des nymphes aux satyres dans un intermède bouffon où Malgrison se révèle un usurier obsédé par les jeunes filles. Paul Vernois cite une lettre de l'auteur où s'affirme la pratique rhapsodique que j'essaie de décrire :

«Je conviens avec vous que l'ardeur de mon héros et les prouesses de Sacripant sont faites pour provoquer quelque surprise. Mais tout cela a été étudié avec soin, pris sur le fait et composé d'enthousiasme. D'ailleurs, je vous le demande, à moins 
d'accoucher d'une berquinade honteuse, pensez-vous qu'il soit possible d'écrire aujourd'hui une idylle où ne se rencontreront pas la peinture franche de la nature et l'accent un peu vif de la passion $^{5}$ ?»

Si l'on se tourne vers Léon Cladel qui est son contemporain du Quercy, on repère la même formule paradoxale. La première partie de L'homme de la Croix-aux-Bøufs ressortit à l'idylle : momentanément en congé du service militaire, Ambroise fait sa cour à Margarido, obtient sa promesse et laisse sa fiancée à la garde de son ami Anzelayr. Or, lorsqu'il se trouve en Afrique, il apprend qu'Anzelayr entreprend d'épouser Margarido. Un tout autre roman se met en place quand Ambroise revient pour se venger. Ambroise gagne la confiance d'Anzelayr, partage ses joyeux banquets pantagruéliques et, finalement, l'ayant fait attaquer par un chien enragé, assiste à son agonie. Le texte le plus célèbre de Léon Cladel est La Fête votive de Saint-Bartholomé-Porte-Glaive (1872). L'auteur y fait alterner l'idylle, l'Iliade et le charivari. Qu'on en juge par ce résumé des premières pages : de retour au pays, l'auteur salue les «grands chênes familiers », le ciel d'enfance, la famille, les ruisseaux. Puis survient une scène réaliste où de joyeux drilles jouent de la flûte et du tambour et vendent des fleurs en papier pour la fête votive du soir. Plus tard, au cours des réjouissances, des charlatans se rassemblent, un clystère farcesque déclenche une guerre picrocholine des Bartholoméens contre les gars de Saint-Carnus. Et Léon Cladel de justifier par une représentation du peuple la formule rhapsodique de son roman : les paysans sont «tour à tour naïfs et subtils, chastes et cyniques, élégiaques et cruels $»$.

Mais les ruptures paradoxales que nous avons observées dans le déroulement d'une œuvre coexistent souvent dans les mêmes lignes d'un texte, dans un mélange d'emphase et de rapetissement. Dans La Fête votive de Saint-Bartholomé-Porte-Glaive un Mage développe un discours ampoulé qui rassemble une puissance barbare qui peut bien stupéfier les paysans : elle n'est pourtant aucunement crédible. Le Mage n'est bien sûr qu'un bonimenteur qui renvoie à Alcofrybas Nasier et au dérèglement des astres qui accompagne la naissance

5. Lettre à M. Templier du 8 mars 1867. V 54. 
de Pantagruel. Écoutons sa poésie cosmique, rustique, et en même temps dévalorisée par son outrance même :

«Oyez, oyez moi ! la lune est rongée par des serpents quarante mille fois plus gros que les vipères de notre sol ; la Charrette (la Petite Ourse) a perdu ses roues, la Poule et ses Poussins (la Grande Ourse) s'en vont, ceux-ci à hue, celle-là à dia ; le Chemin du ciel (la Voie lactée) est effacé, et le Bouc (le Capricorne) ne trouve rien à paitre. Oui ; c'est comme ça. Qu'est-ce que cela signifie? Ah! je ne suis peut-être pas un phénix, moi ; mais puisque vous m'appelez Cent-Yeux, c'est que sans doute vous supposez bien que j'y vois plus finement que ceux qui n'ont à leur service que deux pauvres petites prunelles assez bornées. Eh bien, oui, je sais, moi, ce qui se passe ; et vous voulez le savoir, vous ! Dârdayrael s'exprime, écoutez-le ; il vous instruira... ${ }^{6}$ ».

On pourrait trouver des textes de ce genre dans les $\mathrm{Va}$-nu-pieds où il ne s'agit pas d'un énoncé rapporté mais bien d'un ton assumé par le narrateur qui entrecroise le réalisme, la géorgique et l'épopée :

« Ce soleil rayonnant la vie, sur la terre toujours en travail et toujours en saillie, ces gars maigres et hâlés qui ressembleraient à des moines de Ribeira, si les moines de Ribeira savaient rire, ces vierges augustes comme des druidesses coupant le gui sacré, mais qui ne sont que de belles femmes curieuses et peureuses du mâle ; ces bœufs ruminant solennels et calmes comme des olympiens digérant l'ambroisie; ces ormes et ces châtaigniers bi-séculaires secouant leurs panaches de verdure et faisant danser, comme un voile autour de leurs tronc, l'ombre de leurs bras innombrables ${ }^{7}$ !»

D'où ce jugement de Paul Vernois qui est typique du vertige que produit cette littérature rhapsodique : «Euvre déconcertante, incompréhensible de prime abord, la suite des romans paysans de Cladel a dérouté sinon scandalisé la critique parce qu'elle prodiguait d'un récit à l'autre, voire à l'intérieur d'un même texte, l'injure la

6. Léon Cladel, la Fête votive de Saint-Bartholomé-Porte-Glaive, Paris, A. Lemerre, p. 161.

7. Léon Cladel, Les Va-nu-pieds, Paris, A. Lemerre, 1873, p. 181. 
plus cinglante et la louange la plus dithyrambique à l'adresse de ses personnages ${ }^{8} . »$

Je fais donc l'hypothèse qu'une formule profondément disparate, qui associe parfois séquentiellement et parfois simultanément amplification et diminution du thème, s'installe non seulement dans la même œuvre mais encore dans des unités beaucoup plus réduites, dans le même poème, dans le même chapitre ou dans le même paragraphe. C'est particulièrement le cas dans l'œuvre de Léon Cladel qui fut reçu comme un styliste incongru puisqu'il appliquait son style ciselé à un milieu sordide qu'il se refusait à enjoliver, s'opposant ainsi à une conception catholique et royaliste du paysan. C'est cette contradiction qu'Émile Zola reprochait à Léon Cladel :

«En somme, le grand malheur de M. Léon Cladel est d'être un rhétoricien, un arrangeur de mots. Enfant du Quercy, il a eu l'idée de peindre surtout les paysans au milieu desquels il a grandi. Ce que MM. Erckmann-Chatrian ont fait pour l'Alsace, il le fait pour sa province. Seulement, il y apporte des allures d'épopée. Victor Hugo, avec son style héroïque, a passé par là. Dans le dernier roman que M. Léon Cladel a publié et qui lui a demandé six ans de travail, L'Homme de la Croix-aux-Bœufs, il a eu, ainsi qu 'il l'explique, l'intention de rendre littérairement le langage et les mœurs des paysans du Quercy. Rude besogne et qui ne pouvait aboutir qu 'à une œuvre bâtarde. Cela rappelle la façon dont George Sand faisait parler les paysans du Berry. M. Léon Cladel a plus de vigueur, mais il arrive également à un galimatias poétique. Les paysans ne parlent pas ainsi, ni les poètes non plus ; de sorte que cette langue n'est à personne et qu'elle fatigue horriblement le lecteur au bout de dix pages. Il vaudrait beaucoup mieux étudier les paysans et tâcher de nous les montrer franchement tels qu'ils sont, sans rêver de les rendre littéraires et épiques ${ }^{9}$. »

À l'inverse, Charles Baudelaire repérait la valeur littéraire de cette pratique rhapsodique à propos de son protégé :

« La disproportion du ton avec le sujet, disproportion qui n'est sensible que pour le sage désintéressé, est un moyen de comique

8. Paul Vernois, Le Roman rustique de George Sand à Ramuz : Ses tendances et son évolution, Paris, Nizet, 1962, p. 46.

9. Émile Zola, Les Romanciers Naturalistes, Paris, Charpentier, 1881, p. 370. 
dont la puissance saute à l'œil ; je suis même étonné qu'il ne soit pas employé plus souvent par les peintres de mœurs et les écrivains satiriques, surtout dans les matières concernant l'Amour, véritable magasin de comique peu exploité. Si grand que soit un être, et si nul qu'il soit relativement à l'infini, le pathos et l'emphase lui sont permis et nécessaires : l'Humanité est comme une colonie de ces éphémères de l'Hypanis, dont on a écrit de si jolies fables ; et les fourmis elles-mêmes, pour leurs affaires politiques, peuvent emboucher la trompette de Corneille, proportionnée à leur bouche. Quant aux insectes amoureux, je ne crois pas que les figures de rhétorique dont ils se servent pour gémir leurs passions soient mesquines ; toutes les mansardes entendent tous les soirs des tirades tragiques dont la Comédie-Française ne pourra jamais bénéficier ${ }^{10}$. »

Offrons-nous brièvement deux exemples supplémentaires. Le protagoniste de Tartarin de Tarascon constituera pour nous l'incarnation de ce traitement rhapsodique car il y a explicitement deux Tartarin dans le même homme : le Tartarin Don Quichotte, qui rêve de chasse aux lions et un Tartarin Sancho qui s'inquiète du confort de son ventre. Quant au narrateur, il est constamment dans un lieu interlope, dans une improbable terreur qui dit assez sa distance envers son personnage. Quant à Louis Pergaud, talentueux auteur de La Guerre des boutons, roman de ma douzième année, il revendique dans une lettre de 1912 la formule contradictoire d'une épopée enfantine qui a la forme d'une provocation un peu convenue où l'homme des champs prétend choquer le Parisien dégénéré : «Que cela ne plaise pas aux cervelles légères des boulevardiers qui préfèrent les idylles se déroulant sur les canapés des maisons hospitalières, c'est fort possible, c'est même probable, mais, comme dirait Tailhade : "Ce que j'écris n'est pas pour les charognes !" et j'espère qu'il se trouvera encore un public français - si restreint soit-il - pour goûter le charme de cette épopée ${ }^{11}$.» On sait que cette épopée minuscule s'encanaille dans la trivialité, les mots régionaux et l'influence de Rabelais.

En effet, plus que George Sand jugée trop fade, Rabelais et La Fontaine sont les deux modèles des écrivains régionalistes. Ils

10. Charles Baudelaire, L'Art romantique, Paris, Calmann Levy, 1885.

11. Louis Pergaud, lettre à Edmond Rocher, 18 août 1912, citée par A.-M. THIESSE, op. cit., p. 114. 
leur offrent la caution du prestige et de «l'esprit français» (ce qui n'était pas un critère négligeable au début du $\mathrm{XX}^{\mathrm{e}}$ siècle). Est-il besoin de rappeler que Gargantua raconte une guerre picrocholine qui se déroule dans un espace provincial et limité à quelques lieues ? Ajoutons que l'immense auteur écrit des œuvres dont ni les savants ni le peuple de son temps ne savent exactement que faire : chacun y trouve ce qu'il cherche, mais avec le sentiment de la présence incongrue d'une altérité. Le La Fontaine des Fables est le maître de l'héroï-comique, posant des tragédies et de l'épopée dans le monde minuscule des animaux. Une poule devient une Hélène. Deux coqs au fond d'un poulailler déclenchent une guerre de Troie. On s'avise peu que La Chèvre de M. Seguin est d'abord un apologue qui porte, dans la lignée de La Fontaine, un message de modération: Alphonse Daudet y déconseille à un ami de tenter l'ascension de la montagne du Parnasse. Dans le même temps, tout comme bien des fables, ce conte narre l'inverse de sa moralité déclarée : la quête de l'idéal même au prix de la mort. Quel sujet sublime dans un appel d'humilité !

$\mathrm{Y}$ a-t-il des spécificités régionales dans ce traitement rhapsodique ? Même si le régionalisme est répandu partout ${ }^{12}$, il me semble (dans l'état actuel de mes lectures) que les provinces les moins marquées par les stéréotypes ou par la tradition littéraire rompent avec l'unité de ton en se bornant à donner des gages d'humilité et en justifiant le recours au réalisme par le bon sens du terroir. Par exemple, quand les félibres définissent une littérature auvergnate, c'est simplement par une nuance: «l'exactitude, la précision ingénue, l'émotion sans fracas». Mais les inflexions me paraissent nombreuses dès qu'un légendaire, un grand homme ou un cliché prédominent dans une province. La Touraine, par exemple, peut aisément pratiquer la grivoiserie rabelaisienne, le Nord recourra aux atmosphères brumeuses mises à la mode par Albert Samain. Les Normands peuvent se tourner vers la thématique "Viking" pour assurer la part épique qui s'opposera à l'humilité. Cela produit ces vers au vocabulaire audacieux où Lucie Delarue-Mardrus affirme en même temps un amour des douceurs de la civilisation moderne et la sauvagerie épique d'un pillage de Paris :

12. Sauf en Corse, comme le note Anne-Marie Thiesse. 
«Hors le présent heureux dont mon cœur est épris, Lorsque je vois tomber les couchants équivoques Dans la bénignité de ton fleuve, ô Paris !

Il se réveille en moi - grouillants d'ours et de phoques -

D'agressifs, ancestraux et durs septentrions,

Et des barques blessant la Seine de leurs coques.

Et je crie en mon cœur filial, nous crions

Vers tes mille quartiers, tes palais et tes arches, En préparant nos poings chargés de horions,

Le vent où chantent clair nos gutturales marches Hérisse sur nos caps nos cheveux courts et roux Et nous espérons fort ensanglanter tes marches

Etant d'un terroir plein de ronces et de houx, Où saignent largement les aubes boréales

Et dont les hommes sont brutaux comme des loups ${ }^{13}$.»

On ne sera pas surpris si deux provinces possèdent une forte spécificité rhapsodique. C'est d'abord l'Occitanie qui construit ses stéréotypes sur le prestigieux héritage antique. L'antiquité permet le mélange des tons, d'un côté l'idylle (de Théocrite à Longus), de l'autre l'épopée. Pour les félibres, le peuple provençal serait descendant de Virgile, et ces écrivains confrontent le «ciel latin» à un paysan à la fois violent et truculent, aux désirs exubérants. Dans Ompdrailles de Léon Cladel, il y a résurrection de l'épopée d'Hercule, mais rapetissé dans l'âge moderne. Promenant leur affiche dans les rues, des organisateurs de combats promettent 12 travaux imposés à leur champion. Une fois encore, un bonimenteur construit un discours ampoulé, épique et commercial : "Au temps des Romains et des Grecs, nos antiques modèles, il ne s'est accompli rien de plus chic ni de plus beau. Les grands jours de l'Iliade et de l'Énéide ont reparu. Qu'on y songe. Encelade et les Titans seront dépassés. Un magnifique soleil éclairera ces fêtes homériques. » Bref, Hercule s'y fait Hercule de foire. Quant à la Bretagne régionaliste, elle joue souvent

13. Lucie Delarue-Mardrus, « Marche normande », dans Adolphe van Bever, Les poètes du terroir, Paris, Delagrave, 1909, tome 3. 
dans un même texte sur une quintuple imagerie qui s'est affermie depuis le début du XIX ${ }^{\mathrm{e}}$ siècle. Ces représentations contraignantes en font en même temps soit une terre élégiaque, soit ce que Catherine Bertho ${ }^{14}$ appelle la Bretagne noire, soit un univers druidique, soit au contraire un lieu sordide peint dans un style réaliste, soit enfin, plus rarement, une terre de truculence chafouine.

L'idée d'une pratique de l'écart régionaliste n'est encore qu'une hypothèse de lecture. Du moins considère-t-elle de façon positive une production qui est unanimement rejetée dans le cliché ou dans la maladresse. Elle a donc l'avantage de rendre lisibles certaines œuvres qui ne semblent autrement que risibles. Cette écriture contradictoire des régionalistes est probablement liée à une conception du peuple et de la «grande» littérature : à Paris la littérature harmonieuse, à la province une littérature de l'écart. La province raviverait la fadeur parisienne au moyen d'une sève neuve qui insufflerait dans les thèmes les plus hauts une simplicité nouvelle. Même en se haussant jusqu'à Rome ou jusqu'à Troie, la littérature régionaliste prétend ne pas oublier qu'elle est humble, que ses lieux sont sans prestige, que ses héros ne sont pas vraiment héroïques et qu'elle ne saurait se prendre au sérieux. La rénovation de la littérature française, n'est-ce pas le but que visait Jean Charles-Brun, le porte-parole du mouvement régionaliste dans la première moitié du $\mathrm{XX}^{\mathrm{e}}$ siècle ? Pour lui, la Capitale était capiteuse et ses goûts frelatés, affadis ou grossiers. C'est de l'inspiration régionale qu'il attendait la régénérescence ${ }^{15}$.

14. C. Bertho, «L'invention de la Bretagne - Genèse sociale d'un stéréotype», Actes de la Recherche en Sciences Sociales, $\mathrm{n}^{\circ} 35,1980$, p. 45-62.

15. Jean Charles-Brun, Les Littératures provinciales, Paris, Bloud et Cie, 1907. 